\title{
ATLAS Inner Detector: Commissioning with Cosmics Data
}

\author{
Lucia Masetti* \\ Physikalisches Institut der Universität Bonn, Nußallee 12, 53115 Bonn, Germany \\ E-mail: Lucia.Masetti@cern.ch
}

on behalf of the ATLAS Collaboration

The ATLAS experiment at the CERN Large Hadron Collider (LHC) has started taking data in Autumn 2008 with the inauguration of the LHC. The determination of vertex position and charged particle tracks is performed in the Inner Detector which consists of pixel and microstrip silicon sensors and of straw drift tubes with transition radiation detector capability.

All three subdetectors were run in standalone and also in a combined mode with other ATLAS subsystems. The current status of the three subsystems is reviewed, including results from recent data-taking periods, and from the detector alignment. Particular emphasis is given to the results obtained from cosmic muon runs in preparation for collision data.

European Physical Society Europhysics Conference on High Energy Physics, EPS-HEP 2009,

July 16 - 222009

Krakow, Poland

${ }^{*}$ Speaker. 


\section{The ATLAS Inner Detector}

The ATLAS Inner Detector (ID) [1] combines high-resolution detectors close to the interaction point with continuous tracking elements at the outer radii, all contained in a $2 \mathrm{~T}$ solenoidal field (see Figure 1). The innermost detector is the Pixel Detector, which consists of three layers of silicon modules in the barrel region at a radius of 5,9 and $12 \mathrm{~cm}$, respectively, and three disks in the endcaps. The 1744 modules contain in total 80 million readout channels and cover a sensitive area of $1.6 \mathrm{~m}^{2}$ with $|\eta| \leq 2.5$. The typical pixel dimension is $50 \times 400 \mu \mathrm{m}^{2}$. The Pixel Detector was designed to provide a vertex resolution less than $15 \mu \mathrm{m}$ in the $R \phi$ plane and less than $1 \mathrm{~mm}$ in $z$. In the intermediate region a silicon microstrip detector, called SemiConductor Tracker (SCT), covers radii between 30 and $50 \mathrm{~cm}$. The 4088 SCT modules use a strip pitch of $80 \mu \mathrm{m}$ in the barrel and of 57-90 $\mu \mathrm{m}$ in the endcaps and consist of two sets of sensors glued back-to-back with a stereo angle of $\pm 20 \mathrm{mrad}$. In the barrel region the SCT consists of 4 layers and each endcap contains 9 disks. The outermost part of the ID uses drift (straw) tubes of $4 \mathrm{~mm}$ diameter to provide on average 36 measurements per track at radii from 62 to $107 \mathrm{~cm}$ (barrel) with a position resolution of $130 \mu \mathrm{m}$ in the $R \phi$ plane. The barrel consists of 73 layers of straws and each endcap of 20 wheels with 8 straw layers per wheel. The straw tube detector, called Transition Radiation Tracker (TRT), also provides electron identification through the detection of Transition Radiation photons which are generated when ultra-relativistic particles $\left(\gamma>10^{3}\right)$ traverse the radiator material placed between the straws.

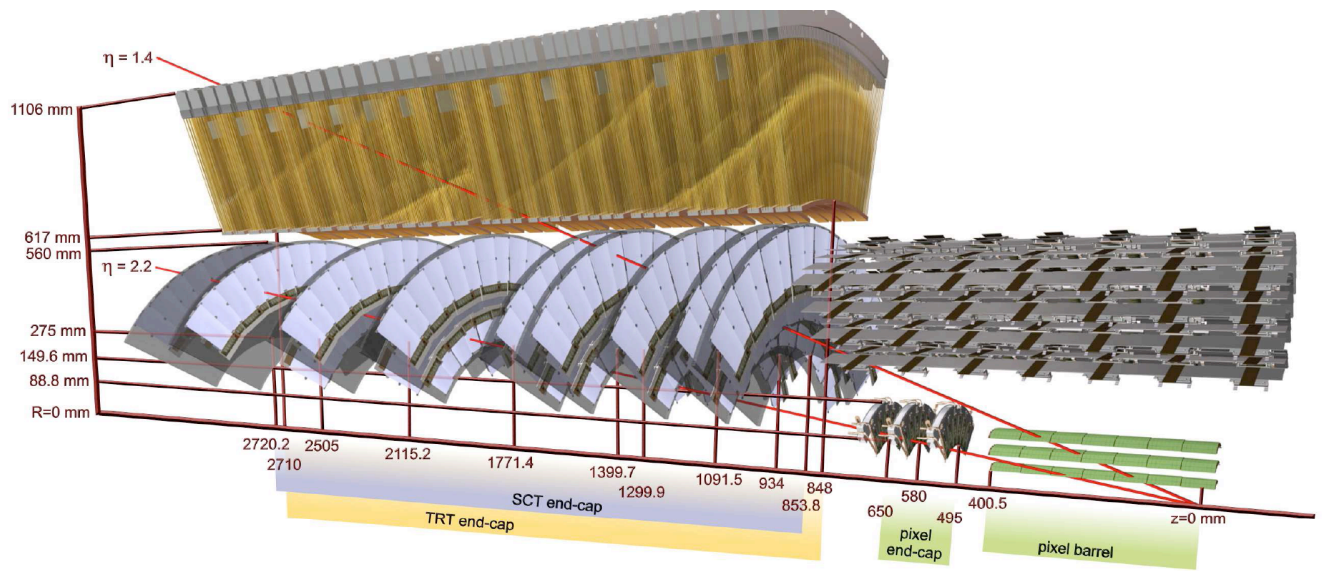

Figure 1: Schematic view of the ATLAS Inner Detector including the barrel layers and endcap disks of Pixel Detector and SCT, and the TRT endcap straw layers.

\section{Commissioning with cosmics data}

After installation in the cavern and the first evaporative cooling commissioning in 2007-2008, the three subdetectors participated in the cosmic muon data taking periods together with the rest of ATLAS starting from September 2008 and were ready for the first LHC beam on September 10th. Starting from November 2008 a TRT-based trigger was used to collect cosmic rays traversing the Inner Detector providing a much higher rate than the muon detectors' trigger used previously. In 2009 evaporative cooling consolidation (affecting Pixel Detector and SCT), standalone TRT tests 

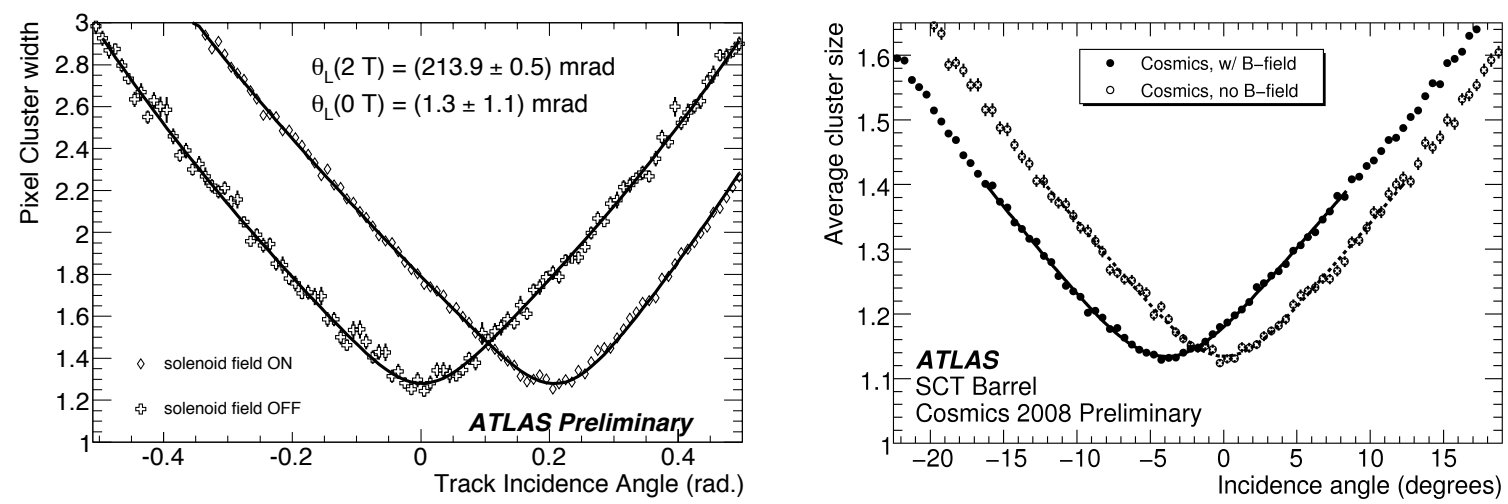

Figure 2: Cluster size vs. track incident angle with and without magnetic field for the Pixel Detector (left) and SCT (right), used to measure the Lorentz angle.

and detector calibration periods were alternated with ATLAS-wide cosmic muon runs. The Inner Detector is now ready for data taking and eagerly waiting for LHC beam. The results reported here were obtained from calibration runs and cosmic ray data taken in 2008 .

At present 2\% of the Pixel Detector cannot be operated due to unrecoverable on-detector failures, mostly caused by the optical transmission or by open HV connections. The innermost layer is affected only to $1.4 \%$. All the cooling loops are working. All the optical transmitter plugins (off-detector) had to be exchanged for ones produced under improved ESD safety conditions. The old ones had a failure rate of a few channels per week of operation. The discriminator threshold was tuned for all the working pixels to $4000 e$, achieving a threshold dispersion of $40 e$. The feedback current of each pixel was tuned such that a minimum ionising particle (mip) results in a Time over Threshold (ToT) of 30 Bunch Crossings (BC) of $25 \mathrm{~ns}$ each. The relationship between deposited charge and ToT was measured up to $40 \mathrm{ke}$, fitted and stored as input for reconstruction and simulation. The noisy channels, corresponding to less than $10^{-4}$ of the total, were masked leading to an average noise occupancy of about $10^{-10}$ hits per BC per pixel. The hit efficiency was measured to be about $99.8 \%$ in the barrel and the alignment resulted in a resolution of $24 \mu \mathrm{m}$ in the $\phi$ direction. The timing resolution was about $6 \mathrm{~ns}$ for high-charge clusters. The Lorentz angle due to the solenoid magnetic field, measured as the track incident angle with the minimum cluster size, was $(213.9 \pm 0.5) \mathrm{mrad}$ (see Figure 2, left).

The SCT uses the same optical transmitter plug-ins as the Pixel Detector, which were exchanged as well. The new plug-ins didn't show any failures since June. One cooling loop in the end-cap cannot be operated, corresponding to $1.3 \%$ of the endcap and $0.35 \%$ of the full detector, while failures due to high or low voltage failures account for a loss of at most $0.6 \%$ of the modules. In the cosmic ray data taken in 2008 the measured noise occupancy was well below the design level of $5 \times 10^{-4}$ hits per BC and the efficiency was between $99 \%$ and $99.5 \%$ in all barrel layers. The Lorentz angle has been measured to $\theta_{L}=\left(3.93 \pm 0.03_{\text {stat }} \pm 0.10_{\text {syst }}\right)$ degrees (see Figure 2, right).

The TRT system commissioning was also successful: the leak rate of the active gas system in the TRT was reduced to $0.15 \mathrm{l} / \mathrm{h}$, which is the lowest leak rate of any large volume detector in the LHC, while the non-recoverable failures of detector parts amount to $2.2 \%$ of the straws being dead. The TRT noise occupancy is typically of the order of $2 \%$. During cosmic rays data taking the straw tube efficiency was measured to be $(97.2 \pm 0.5) \%$. The high-threshold hit probability as 


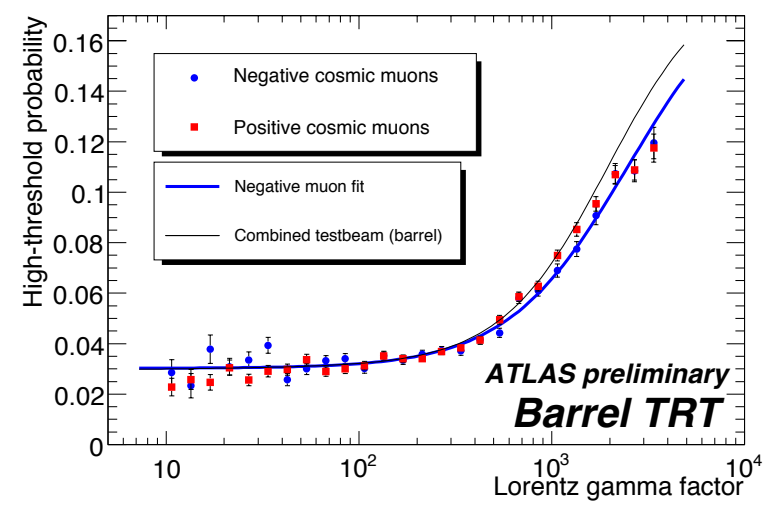

Figure 3: Transition radiation onset in the barrel TRT.
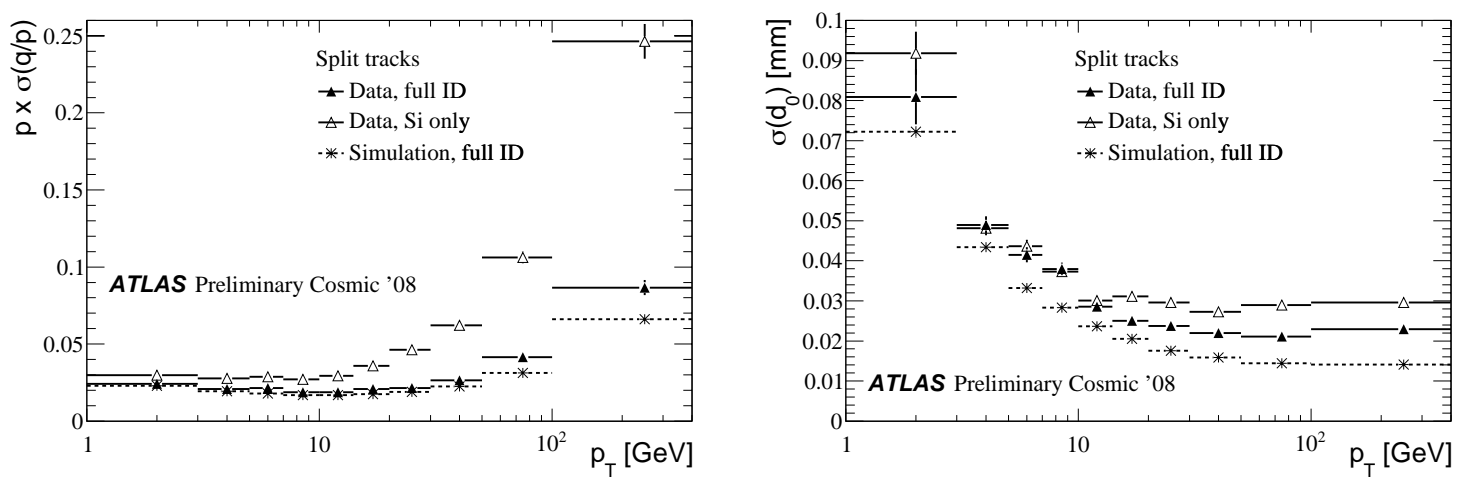

Figure 4: Momentum (left) and impact parameter (right) resolution as a function of the transverse momentum, obtained from cosmic muon data.

a function of the Lorentz $\gamma$ factor of the incident particle confirmed the results from previous tests (see Figure 3).

Cosmic ray tracks that cross both the upper and the lower hemisphere of the ID can be split in the centre and are refitted separately to measure the track parameter resolution and bias directly from the data (see Figure 4). The alignment obtained from cosmic ray data already achieved a resolution very close to the ideal detector performace, while the residual small biases are currently under study.

\section{Summary and outlook}

At present $98 \%$ of the Pixel Detector, $99.5 \%$ of SCT and $98 \%$ of TRT are operational. The cosmic ray data have been very valuable to understand the detector performances. The silicon detectors' noise occupancy and hit efficiency are better than the specifications. The TRT has been working very reliably for a long time and is providing the timing for triggering on cosmic ray tracks and the track parameter resolution is already close to expectation from the simulation of the perfect detector. The ATLAS Inner Detector is well prepared for first collisions.

\section{References}

[1] G. Aad et al. [ATLAS Collaboration], "The ATLAS Experiment at the CERN Large Hadron Collider," JINST 3 (2008) S08003. 